Geometry ${ }^{6}$ Topology

Volume 1 (1997) 51-69

Published: 26 October 1997

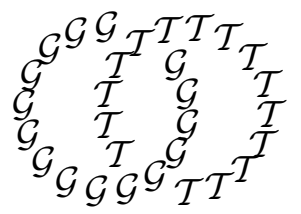

\title{
Alexander Duality, Gropes and Link Homotopy
}

\author{
VyACHESLAV S KRUSHKAL \\ Peter Teichner \\ Department of Mathematics, Michigan State University \\ East Lansing, MI 48824-1027, USA \\ Current address: Max-Planck-Institut für Mathematik \\ Gottfried-Claren-Strasse 26, D-53225 Bonn, Germany \\ and \\ Department of Mathematics \\ University of California in San Diego \\ La Jolla, CA, 92093-0112, USA \\ Email: krushkal@math.msu.edu and teichner@euclid.ucsd.edu
}

\begin{abstract}
We prove a geometric refinement of Alexander duality for certain 2-complexes, the so-called gropes, embedded into 4-space. This refinement can be roughly formulated as saying that 4-dimensional Alexander duality preserves the disjoint Dwyer filtration.

In addition, we give new proofs and extended versions of two lemmas of Freedman and Lin which are of central importance in the $A$-B-slice problem, the main open problem in the classification theory of topological 4-manifolds. Our methods are group theoretical, rather than using Massey products and Milnor $\mu$-invariants as in the original proofs.
\end{abstract}

AMS Classification numbers Primary: 55M05, 57M25

Secondary: $57 \mathrm{M} 05,57 \mathrm{~N} 13,57 \mathrm{~N} 70$

Keywords: Alexander duality, 4-manifolds, gropes, link homotopy, Milnor group, Dwyer filtration

Proposed: Robion Kirby

Received: 17 June 1997

Seconded: Michael Freedman, Ronald Stern

Revised: 17 October 1997

Copyright Geometry and Topology 


\section{Introduction}

Consider a finite complex $X$ PL-embedded into the $n$-dimensional sphere $S^{n}$. Alexander duality identifies the (reduced integer) homology $H_{i}\left(S^{n} \backslash X\right)$ with the cohomology $H^{n-1-i}(X)$. This implies that the homology (or even the stable homotopy type) of the complement cannot distinguish between possibly different embeddings of $X$ into $S^{n}$. Note that there cannot be a duality for homotopy groups as one can see by considering the fundamental group of classical knot complements, ie the case $X=S^{1}$ and $n=3$.

However, one can still ask whether additional information about $X$ does lead to additional information about $S^{n} \backslash X$. For example, if $X$ is a smooth closed $(n-$ $1-i$ )-dimensional manifold then the cohomological fundamental class is dual to a spherical class in $H_{i}\left(S^{n} \backslash X\right)$. Namely, it is represented by any meridional $i$-sphere which by definition is the boundary of a normal disk at a point in $X$. This geometric picture explains the dimension shift in the Alexander duality theorem.

By reversing the roles of $X$ and $S^{n} \backslash X$ in this example we see that it is not true that $H_{i}(X)$ being spherical implies that $H_{n-1-i}\left(S^{n} \backslash X\right)$ is spherical. However, the following result shows that there is some kind of improved duality if one does not consider linking dimensions. One should think of the Gropes in our theorem as means of measuring how spherical a homology class is.

Theorem 1 (Grope Duality) If $X \subset S^{4}$ is the disjoint union of closed embedded Gropes of class $k$ then $\mathrm{H}_{2}\left(S^{4} \backslash X\right)$ is freely generated by $r$ disjointly embedded closed Gropes of class $k$. Here $r$ is the rank of $H_{1}(X)$. Moreover, $H_{2}\left(S^{4} \backslash X\right)$ cannot be generated by $r$ disjoint maps of closed gropes of class $k+1$.

As a corollary to this result we show in 4.2 that certain Milnor $\mu$-invariants of a link in $S^{3}$ are unchanged under a Grope concordance.

The Gropes above are framed thickenings of very simple 2-complexes, called gropes, which are inductively built out of surface stages, see Figure 1 and Section 2. For example, a grope of class 2 is just a surface with a single boundary component and gropes of bigger class contain information about the lower central series of the fundamental group. Moreover, every closed grope has a fundamental class in $H_{2}(X)$ and one obtains a geometric definition of the Dwyer filtration

$$
\pi_{2}(X) \subseteq \ldots \subseteq \phi_{k}(X) \subseteq \ldots \subseteq \phi_{3}(X) \subseteq \phi_{2}(X)=H_{2}(X)
$$

\section{Geometry and Topology, Volume 1 (1997)}


by defining $\phi_{k}(X)$ to be the set of all homology classes represented by maps of closed gropes of class $k$ into $X$. Theorem 1 can thus be roughly formulated as saying that 4-dimensional Alexander duality preserves the disjoint Dwyer filtration.
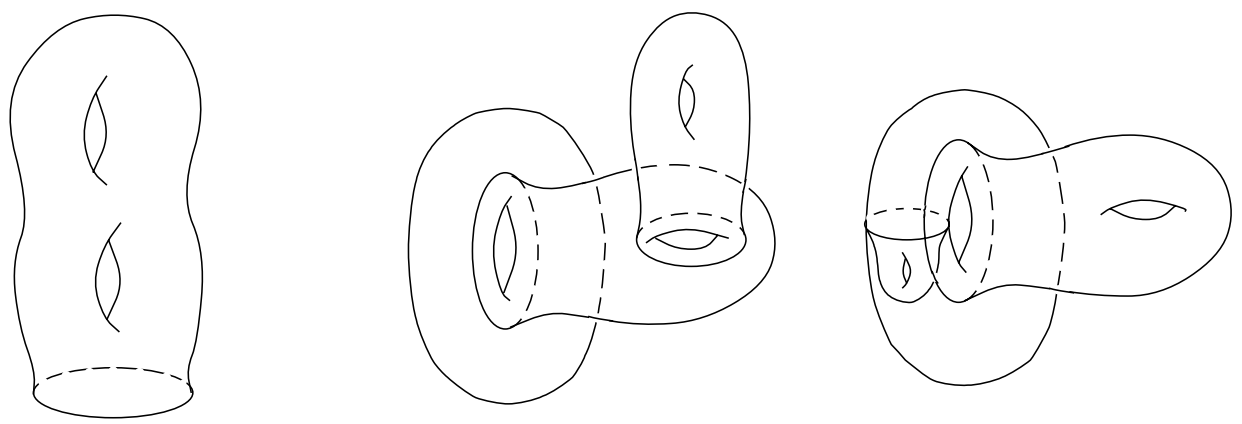

Figure 1: A grope of class 2 is a surface - two closed gropes of class 4

Figure 1 shows that each grope has a certain "type" which measures how the surface stages are attached. In Section 2 this will be made precise using certain rooted trees, compare Figure 2. In Section 4 we give a simple algorithm for obtaining the trees corresponding to the dual Gropes constructed in Theorem 1.

The simplest application of Theorem 1 (with class $k=2$ ) is as follows. Consider the standard embedding of the 2 -torus $T^{2}$ into $S^{4}$ (which factors through the usual unknotted picture of $T^{2}$ in $S^{3}$ ). Then the boundary of the normal bundle of $T^{2}$ restricted to the two essential circles gives two disjointly embedded tori representing generators of $H_{2}\left(S^{4} \backslash T^{2}\right) \cong \mathbb{Z}^{2}$. Since both of these tori may be surgered to (embedded) spheres, $H_{2}\left(S^{4} \backslash T^{2}\right)$ is in fact spherical. However, it cannot be generated by two maps of 2 -spheres with disjoint images, since a map of a sphere may be replaced by a map of a grope of arbitrarily big class.

This issue of disjointness leads us to study the relation of gropes to classical link homotopy. We use Milnor group techniques to give new proofs and improved versions of the two central results of [2], namely the Grope Lemma and the Link Composition Lemma. Our generalization of the grope lemma reads as follows.

Theorem 2 Two $n$-component links in $S^{3}$ are link homotopic if and only if they cobound disjointly immersed annulus-like gropes of class $n$ in $S^{3} \times I$.

This result is stronger than the version given in [2] where the authors only make a comparison with the trivial link. Moreover, our new proof is considerably shorter than the original one.

Geometry and Topology, Volume 1 (1997) 
Our generalization of the link composition lemma is formulated as Theorem 3 in Section 5. The reader should be cautious about the proof given in [2]. It turns out that our Milnor group approach contributes a beautiful feature to Milnor's algebraization of link homotopy: He proved in [10] that by forgetting one component of the unlink one gets an abelian normal subgroup of the Milnor group which is the additive group of a certain ring $R$. We observe that the Magnus expansion of the free Milnor groups arises naturally from considering the conjugation action of the quotient group on this ring $R$. Moreover, we show in Lemma 5.3 that "composing" one link into another corresponds to multiplication in that particular ring $R$. This fact is the key in our proof of the link composition lemma.

Our proofs completely avoid the use of Massey products and Milnor $\mu$-invariants and we feel that they are more geometric and elementary than the original proofs. This might be of some use in studying the still unsolved $A$ - $B$-slice problem which is the main motivation behind trying to relate gropes, their duality and link homotopy. It is one form of the question whether topological surgery and s-cobordism theorems hold in dimension 4 without fundamental group restrictions. See [4] for new developments in that area.

Acknowledgements: It is a pleasure to thank Mike Freedman for many important discussions and for providing an inspiring atmosphere in his seminars. In particular, we would like to point out that the main construction of Theorem 1 is reminiscent of the methods used in the linear grope height raising procedure of [5]. The second author would like to thank the Miller foundation at UC Berkeley for their support.

\section{Preliminary facts about gropes and the lower central series}

The following definitions are taken from [5].

Definition 2.1 A grope is a special pair (2-complex, circle). A grope has a class $k=1,2, \ldots, \infty$. For $k=1$ a grope is defined to be the pair (circle, circle). For $k=2$ a grope is precisely a compact oriented surface $\Sigma$ with a single boundary component. For $k$ finite a $k$-grope is defined inductively as follow: Let $\left\{\alpha_{i}, \beta_{i}, i=1, \ldots\right.$, genus $\}$ be a standard symplectic basis of circles for $\Sigma$. For any positive integers $p_{i}, q_{i}$ with $p_{i}+q_{i} \geq k$ and $p_{i_{0}}+q_{i_{0}}=k$ for at least one index $i_{0}$, a $k$-grope is formed by gluing $p_{i}$-gropes to each $\alpha_{i}$ and $q_{i}$-gropes to each $\beta_{i}$.

Geometry and Topology, Volume 1 (1997) 
The important information about the "branching" of a grope can be very well captured in a rooted tree as follows: For $k=1$ this tree consists of a single vertex $v_{0}$ which is called the root. For $k=2$ one adds $2 \cdot$ genus $(\Sigma)$ edges to $v_{0}$ and may label the new vertices by $\alpha_{i}, \beta_{i}$. Inductively, one gets the tree for a $k$-grope which is obtained by attaching $p_{i}$-gropes to $\alpha_{i}$ and $q_{i}-$ gropes to $\beta_{i}$ by identifying the roots of the $p_{i}$-(respectively $q_{i}-$ )gropes with the vertices labeled by $\alpha_{i}$ (respectively $\beta_{i}$ ). Figure 2 below should explain the correspondence between gropes and trees.
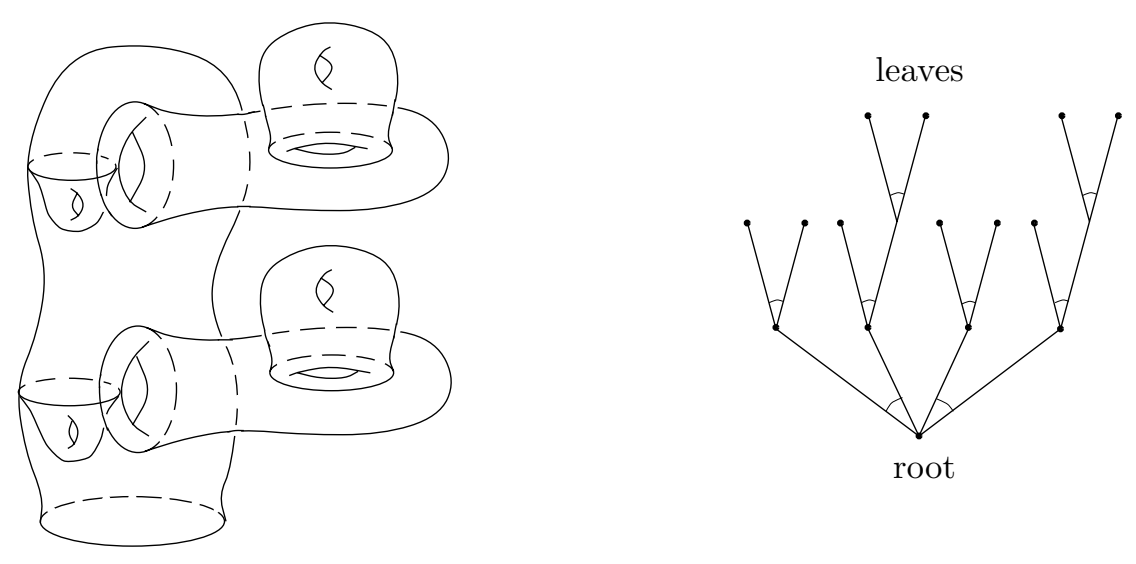

Figure 2: A grope of class 5 and the associated tree

Note that the vertices of the tree which are above the root $v_{0}$ come in pairs corresponding to the symplectic pairs of circles in a surface stage and that such rooted paired trees correspond bijectively to gropes. Under this bijection, the leaves $(:=1$-valent vertices) of the tree correspond to circles on the grope which freely generate its fundamental group. We will sometimes refer to these circles as the tips of the grope. The boundary of the first stage surface $\Sigma$ will be referred to as the bottom of the grope.

Given a group $\Gamma$, we will denote by $\Gamma^{k}$ the $k$-th term in the lower central series of $\Gamma$, defined inductively by $\Gamma^{1}:=\Gamma$ and $\Gamma^{k}:=\left[\Gamma, \Gamma^{k-1}\right]$, the characteristic subgroup of $k$-fold commutators in $\Gamma$.

Lemma 2.2 (Algebraic interpretation of gropes [5, 2.1]) For a space $X$, a loop $\gamma$ lies in $\pi_{1}(X)^{k}, 1 \leq k<\omega$, if and only if $\gamma$ bounds a map of some $k$-grope. Moreover, the class of a grope $(G, \gamma)$ is the maximal $k$ such that $\gamma \in \pi_{1}(G)^{k}$.

A closed $k$-grope is a 2 -complex made by replacing a 2 -cell in $S^{2}$ with a $k-$ grope. A closed grope is sometimes also called a sphere-like grope. Similarly,

Geometry and Topology, Volume 1 (1997) 
one has annulus-like $k$-gropes which are obtained from an annulus by replacing a 2 -cell with a $k$-grope. Given a space $X$, the Dwyer's subgroup $\phi_{k}(X)$ of $H_{2}(X)$ is the set of all homology classes represented by maps of closed gropes of class $k$ into $X$. Compare $[5,2.3]$ for a translation to Dwyer's original definition.

Theorem (Dwyer's Theorem [1]) Let $k$ be a positive integer and let $f$ : $X \longrightarrow Y$ be a map inducing an isomorphism on $H_{1}$ and an epimorphism on $H_{2} / \phi_{k}$. Then $f$ induces an isomorphism on $\pi_{1} /\left(\pi_{1}\right)^{k}$.

A Grope is a special "untwisted" 4-dimensional thickening of a grope $(G, \gamma)$; it has a preferred solid torus (around the base circle $\gamma$ ) in its boundary. This "untwisted" thickening is obtained by first embedding $G$ in $\mathbb{R}^{3}$ and taking its thickening there, and then crossing it with the interval $[0,1]$. The definition of a Grope is independent of the chosen embedding of $G$ in $\mathbb{R}^{3}$. One can alternatively define it by a thickening of $G$ such that all relevant relative Euler numbers vanish. Similarly, one defines sphere- and annulus-like Gropes, the capital letter indicating that one should take a 4-dimensional untwisted thickening of the corresponding $2-$ complex.

\section{The Grope Lemma}

We first recall some material from [10]. Two $n$-component links $L$ and $L^{\prime}$ in $S^{3}$ are said to be link-homotopic if they are connected by a 1-parameter family of immersions such that distinct components stay disjoint at all times. $L$ is said to be homotopically trivial if it is link-homotopic to the unlink. $L$ is almost homotopically trivial if each proper sublink of $L$ is homotopically trivial.

For a group $\pi$ normally generated by $g_{1}, \ldots, g_{k}$ its Milnor group $M \pi$ (with respect to $g_{1}, \ldots, g_{k}$ ) is defined to be the quotient of $\pi$ by the normal subgroup generated by the elements $\left[g_{i}, g_{i}^{h}\right]$, where $h \in \pi$ is arbitrary. Here we use the conventions

$$
\left[g_{1}, g_{2}\right]:=g_{1} \cdot g_{2} \cdot g_{1}^{-1} \cdot g_{2}^{-1} \text { and } g^{h}:=h^{-1} \cdot g \cdot h .
$$

$M \pi$ is nilpotent of class $\leq k+1$, ie it is a quotient of $\pi /(\pi)^{k+1}$, and is generated by the quotient images of $g_{1}, \ldots, g_{k}$, see [4]. The Milnor group $M(L)$ of a link $L$ is defined to be $M \pi_{1}\left(S^{3} \backslash L\right)$ with respect to its meridians $m_{i}$. It is the largest common quotient of the fundamental groups of all links link-homotopic to $L$, hence one obtains:

Geometry and Topology, Volume 1 (1997) 
Theorem (Invariance under link homotopy [10]) If $L$ and $L^{\prime}$ are link homotopic then their Milnor groups are isomorphic.

The track of a link homotopy in $S^{3} \times I$ gives disjointly immersed annuli with the additional property of being mapped in a level preserving way. However, this is not really necessary for $L$ and $L^{\prime}$ to be link homotopic, as the following result shows.

Lemma 3.1 (Singular concordance implies homotopy [6], [7], [9]) If $L \subset S^{3} \times\{0\}$ and $L^{\prime} \subset S^{3} \times\{1\}$ are connected in $S^{3} \times I$ by disjointly immersed annuli then $L$ and $L^{\prime}$ are link-homotopic.

Remark This result was recently generalized to all dimensions, see [13].

Our Grope Lemma (Theorem 2 in the introduction) further weakens the conditions on the objects that connect $L$ and $L^{\prime}$.

Proof of Theorem 2 Let $G_{1}, \ldots, G_{n}$ be disjointly immersed annulus-like gropes of class $n$ connecting $L$ and $L^{\prime}$ in $S^{3} \times I$. To apply the above Lemma 3.1, we want to replace one $G_{i}$ at a time by an immersed annulus $A_{i}$ in the complement of all gropes and annuli previously constructed.

Let's start with $G_{1}$. Consider the circle $c_{1}$ which consists of the union of the first component $l_{1}$ of $L$, then an arc in $G_{1}$ leading from $l_{1}$ to $l_{1}^{\prime}$, then the first component $l_{1}^{\prime}$ of $L^{\prime}$ and finally the same arc back to the base point. Then the $n$-grope $G_{1}$ bounds $c_{1}$ and thus $c_{1}$ lies in the $n$-th term of the lower central series of the group $\pi_{1}\left(S^{3} \times I \backslash G\right)$, where $G$ denotes the union of $G_{2}, \ldots, G_{n}$. As first observed by Casson, one may do finitely many finger moves on the bottom stage surfaces of $\mathrm{G}$ (keeping the components $G_{i}$ disjoint) such that the natural projection induces an isomorphism

$$
\pi_{1}\left(S^{3} \times I \backslash G\right) \cong M \pi_{1}\left(S^{3} \times I \backslash G\right)
$$

(see [4] for the precise argument, the key idea being that the relation $\left[m_{i}, m_{i}^{h}\right]$ can be achieved by a self finger move on $G_{i}$ which follows the loop $h$.) But the latter Milnor group is normally generated by $(n-1)$ meridians and is thus nilpotent of class $\leq n$. In particular, $c_{i}$ bounds a disk in $S^{3} \times I \backslash G$ which is equivalent to saying that $l_{1}$ and $l_{1}^{\prime}$ cobound an annulus $A_{1}$, disjoint from $G_{2}, \ldots, G_{n}$.

Since finger moves only change the immersions and not the type of a 2-complex, ie an immersed annulus stays an immersed annulus, the above argument can be repeated $n$ times to get disjointly immersed annuli $A_{1}, \ldots, A_{n}$ connecting $L$ and $L^{\prime}$.

Geometry and Topology, Volume 1 (1997) 


\section{Grope Duality}

In this section we give the proof of Theorem 1 and a refinement which explains what the trees corresponding to the dual Gropes look like. Since we now consider closed gropes, the following variation of the correspondence to trees turns out to be extremely useful. Let $G$ be a closed grope and let $G^{\prime}$ denote $G$ with a small 2-cell removed from its bottom stage. We define the tree $T_{G}$ to be the tree corresponding to $G^{\prime}$ (as defined in Section 2) together with an edge added to the root vertex. This edge represents the deleted 2 -cell and it turns out to be useful to define the root of $T_{G}$ to be the 1 -valent vertex of this new edge. See Figure 4 for an example of such a tree.

Proof of Theorem 1 Abusing notation, we denote by $X$ the core grope of the given 4 -dimensional Grope in $S^{4}$. Thus $X$ is a 2 -complex which has a particularly simple thickening in $S^{4}$ which we may use as a regular neighborhood. All constructions will take place in this regular neighborhood, so we may assume that $X$ has just one connected component. Let $\left\{\alpha_{i, j}, \beta_{i, j}\right\}$ denote a standard symplectic basis of curves for the $i$-th stage $X_{i}$ of $X$; these curves correspond to vertices at a distance $i+1$ from the root in the associated tree. Here $X_{1}$ is the bottom stage and thus a closed connected surface. For $i>1$, the $X_{i}$ are disjoint unions of punctured surfaces. They are attached along some of the curves $\alpha_{i-1, j}$ or $\beta_{i-1, j}$.

Let $A_{i, j}$ denote the $\epsilon$-circle bundle of $X_{i}$ in $S^{4}$, restricted to a parallel displacement of $\alpha_{i, j}$ in $X_{i}$, see Figure 3. The corresponding $\epsilon$-disk bundle, for

$\epsilon$ small enough, can be used to see that the 2 -torus $A_{i, j}$ has linking number 1 with $\beta_{i, j}$ and does not link other curves in the collection $\left\{\alpha_{s, t}, \beta_{s, t}\right\}$. Note that if there is a higher stage attached to $\beta_{i, j}$ then it intersects $A_{i, j}$ in a single point, while if there is no stage attached to $\beta_{i, j}$ then $A_{i, j}$ is disjoint from $X$, and the generator of $H_{2}\left(S^{4} \backslash X\right)$ represented by $A_{i, j}$ is Alexander-dual to $\beta_{i, j}$. Similarly, let $B_{i, j}$ denote a 2 -torus representative of the class dual to $\alpha_{i, j}$. There are two inductive steps used in the construction of the stages of dual Gropes.

Step 1 Let $\gamma$ be a curve in the collection $\left\{\alpha_{i, j}, \beta_{i, j}\right\}$, and let $X^{\prime}$ denote the subgrope of $X$ which is attached to $\gamma$. Since $X$ is framed and embedded, a parallel copy of $\gamma$ in $S^{4}$ bounds a parallel copy of $X^{\prime}$ in the complement of $X$. If there is no higher stage attached to $\gamma$ then the application of Step 1 to this curve is empty.

Step 2 Let $\Sigma_{i}$ be a connected component of the $i$-th stage of $X$, and let $m_{i}$ denote a meridian of $\Sigma_{i}$ in $S^{4}$, that is, $m_{i}$ is the boundary of a small normal 

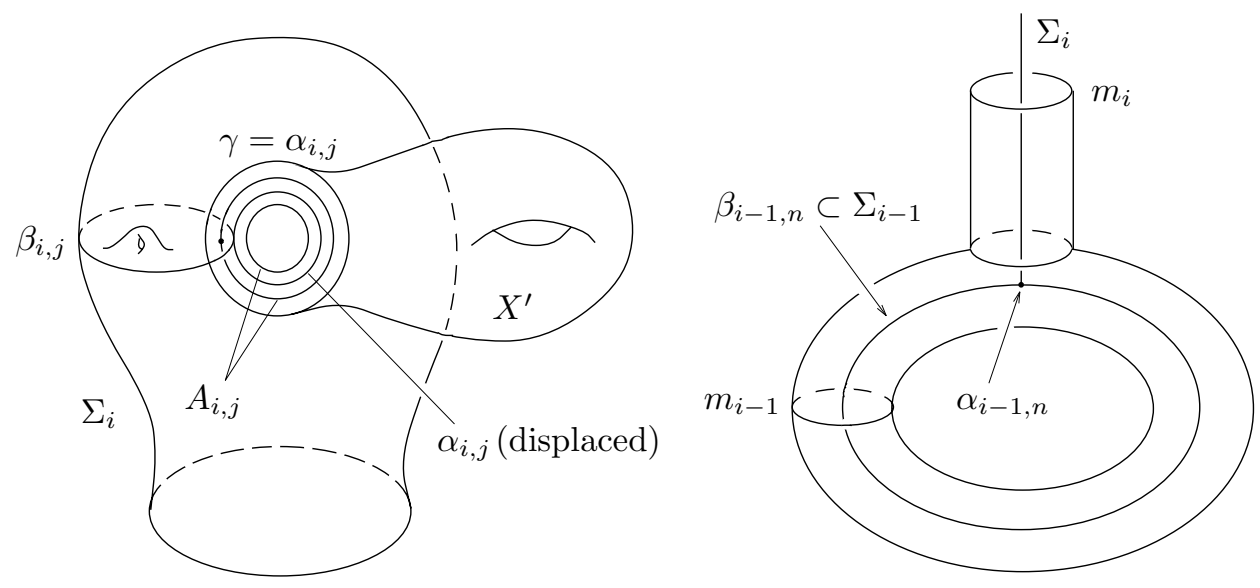

Figure 3: Steps 1 and 2

disk to $\Sigma_{i}$ at an interior point. Suppose $i>1$ and let $\Sigma_{i-1}$ denote the previous stage, so that $\Sigma_{i}$ is attached to $\Sigma_{i-1}$ along some curve, say $\alpha_{i-1, n}$. The torus $B_{i-1, n}$ meets $\Sigma_{i}$ in a point, but making a puncture into $B_{i-1, n}$ around this intersection point and connecting it by a tube with $m_{i}$ exhibits $m_{i}$ as the boundary of a punctured torus in the complement of $X$, see Figure 3 .

By construction, $H_{1}(X)$ is generated by those curves $\left\{\alpha_{i, j}, \beta_{i, j}\right\}$ which do not have a higher stage attached to them. Fix one of these curves, say $\beta_{i, j}$. We will show that its dual torus $A_{i, j}$ is the first stage of an embedded Grope $G \subset S^{4} \backslash X$ of class $k$. The meridian $m_{i}$ and a parallel copy of $\alpha_{i, j}$ form a symplectic basis of circles for $A_{i, j}$. Apply Step 1 to $\alpha_{i, j}$. If $i=1$, the result of Step 1 is a grope at least of class $k$ and we are done. If $i>1$, apply in addition Step 2 to $m_{i}$. The result of Step 2 is a grope with a new genus 1 surface stage, the tips of which are the meridian $m_{i-1}$ and a parallel copy of one of the standard curves in the previous stage, say $\beta_{i-1, n}$. The next Step 1 - Step 2 cycle is applied to these tips. Altogether there are $i$ cycles, forming the grope $G$.

The trees corresponding to dual gropes constructed above may be read off the tree associated to $X$, as follows. Start with the tree $T_{X}$ for $X$, and pick the tip (1-valent vertex), corresponding to the curve $\beta_{i, j}$. The algorithm for drawing the tree $T_{G}$ of the grope $G$, Alexander-dual to $\beta_{i, j}$, reflects Steps 1 and 2 above. Consider the path $p$ from $\beta_{i, j}$ to the root of $T_{X}$, and start at the vertex $\alpha_{i-1, n}$, adjacent to $\beta_{i, j}$. Erase all branches in $T_{X}$, "growing" from $\alpha_{i-1, n}$, except for the edge $\left[\beta_{i, j} \alpha_{i-1, n}\right]$ which has been previously considered, and its "partner" branch $\left[\alpha_{i, j} \alpha_{i-1, n}\right]$, and then move one edge down along the path $p$. This step 
is repeated $i$ times, until the root of $T_{X}$ is reached. The tree $T_{G}$ is obtained by copying the part of $T_{X}$ which is not erased, with the tip $\beta_{i, j}$ drawn as the root, see figure 4 .
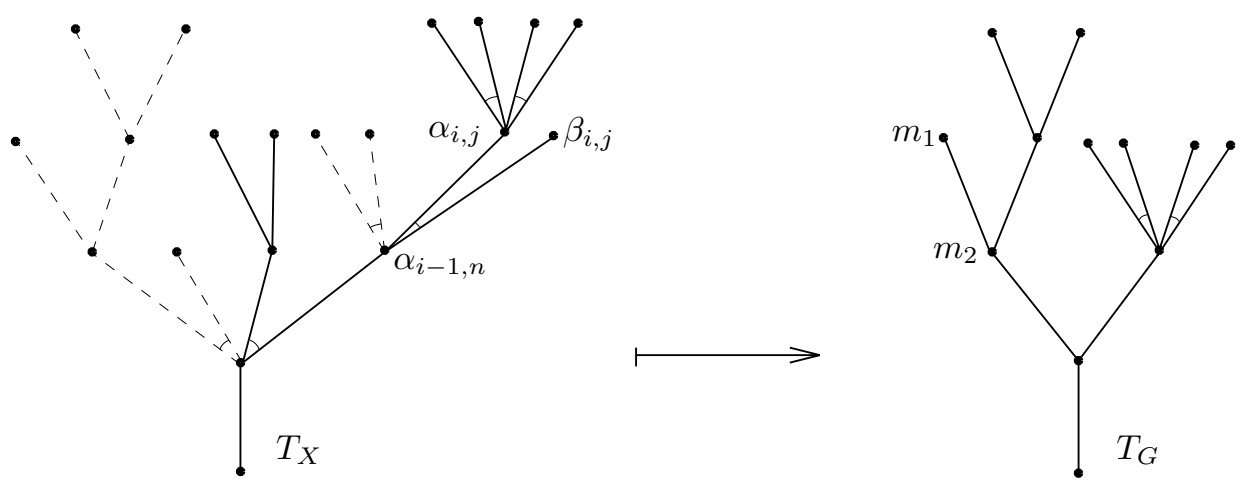

Figure 4: A dual tree: The branches in $T_{X}$ to be erased are drawn with dashed lines.

Note the "distinguished" path in $T_{G}$, starting at the root and labelled by $m_{i}, m_{i-1}, \ldots, m_{1}$. Each of the vertices $m_{i}, m_{i-1}, \ldots, m_{2}$ is trivalent (this corresponds to the fact that all surfaces constructed by applications of Step 2 have genus 1 ), see figures 4,6 . In particular, the class of $G$ may be computed as the sum of classes of the gropes attached to the "partner" vertices of $m_{i}, \ldots, m_{1}$, plus 1 .

We will now prove that the dual grope $G$ is at least of class $k$. The proof is by induction on the class of $X$. For surfaces (class $=2$ ) the construction gives tori in the collection $\left\{A_{i, j}, B_{i, j}\right\}$. Suppose the statement holds for Gropes of class less than $k$, and let $X$ be a Grope of class $k$. By definition, for each standard pair of dual circles $\alpha, \beta$ in the first stage $\Sigma$ of $X$ there is a $p$-grope $X_{\alpha}$ attached to $\alpha$ and a $q$-grope $X_{\beta}$ attached to $\beta$ with $p+q \geq k$. Let $\gamma$ be one of the tips of $X_{\alpha}$. By the induction hypothesis, the grope $G_{\alpha}$ dual to $\gamma$, given by the construction above for $X_{\alpha}$, is at least of class $p . G$ is obtained from $G_{\alpha}$ by first attaching a genus 1 surface to $m_{2}$, with new tips $m_{1}$ and a parallel copy of $\beta$ (Step 2), and then attaching a parallel copy of $X_{\beta}$ (Step 1). According to the computation above of the class of $G$ in terms of its tree, it is equal to $p+q \geq k$.

It remains to show that the dual gropes can be made disjoint, and that they are 0 -framed. Each dual grope may be arranged to lie in the boundary of a regular $\epsilon$-neighborhood of $X$, for some small $\epsilon$. Figure 5 shows how Steps 1 and 2 are performed at a distance $\epsilon$ from $X$. Note that although tori $A_{i, j}$ and 
$B_{i, j}$ intersect, at most one of them is used in the construction of a dual grope for each index $(i, j)$. Taking distinct values $\epsilon_{1}, \ldots, \epsilon_{r}$, the gropes are arranged to be pairwise disjoint. The same argument shows that each grope $G$ has a parallel copy $G^{\prime}$ with $G \cap G^{\prime}=\emptyset$, hence its thickening in $S^{4}$ is standard.

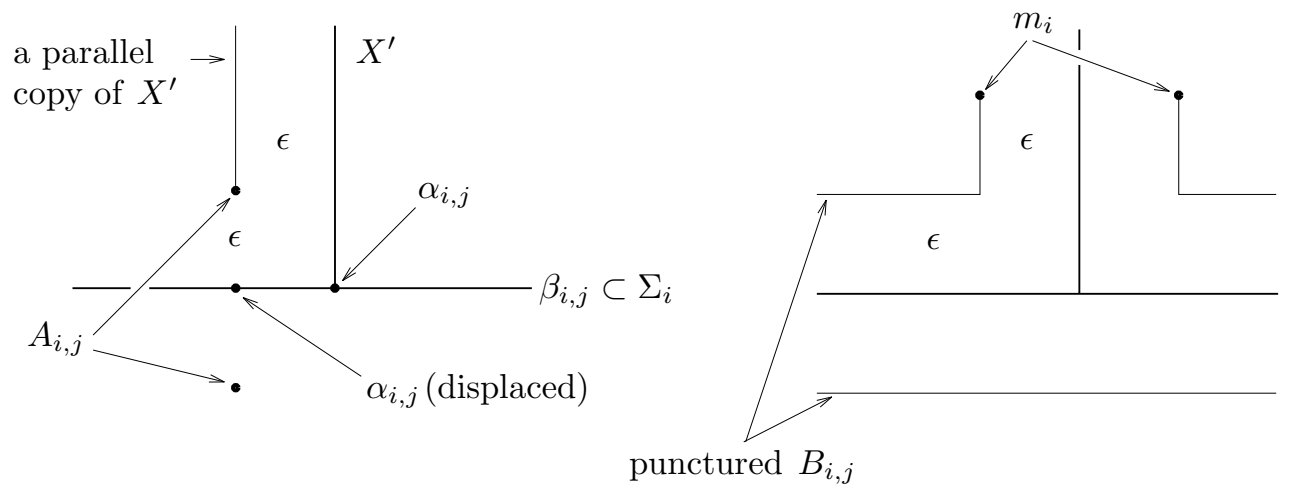

Figure 5: Steps 1 and 2 at a distance $\epsilon$ from $X$

To prove the converse part of the theorem, suppose that $H_{2}\left(S^{4} \backslash X\right)$ is generated by $r$ disjoint maps of closed gropes. Perturb the maps in the complement of $X$, so that they are immersions and their images have at most a finite number of transverse self-intersection points. The usual pushing down and twisting procedures from [3] produce closed disjoint 0 -framed gropes $G_{1}, \ldots, G_{r}$ whose only failure to being actual Gropes lies in possible self-intersections of the bottom stage surfaces. The $G_{i}$ still lie in the complement of $X$ and have class $k+1$. The proof of the first part of Theorem 1 shows that $H_{2}(Y) / \phi_{k+1}(Y)$ is generated by the "Clifford tori" in the neighborhoods of self-intersection points of the $G_{i}$, where $Y$ denotes the complement of all $G_{i}$ in $S^{4}$. Assume $X$ is connected (otherwise consider a connected component of $X$ ), and let $X^{\prime}$ denote $X$ with a 2-cell removed from its bottom stage. The relations given by the Clifford tori are among the defining relations of the Milnor group on meridians to the gropes, and Dwyer's theorem shows (as in [5], Lemma 2.6) that the inclusion map induces an isomorphism

$$
M \pi_{1}\left(X^{\prime}\right) / M \pi_{1}\left(X^{\prime}\right)^{k+1} \cong M \pi_{1}(Y) / M \pi_{1}(Y)^{k+1} .
$$

Consider the boundary curve $\gamma$ of $X^{\prime}$. Since $X$ is a grope of class $k$, by Lemma 4.1 below we get $\gamma \notin M \pi_{1}\left(X^{\prime}\right)^{k+1}$. On the other hand, $\gamma$ bounds a disk in $Y$, hence $\gamma=1 \in M \pi_{1}(Y)$. This contradiction concludes the proof of Theorem 1.

Lemma 4.1 Let $(G, \gamma)$ be a grope of class $k$. Then $\gamma \notin M \pi_{1}(G)^{k+1}$. 
Proof This is best proven by an induction on $k$, starting with the fact that $\pi_{1}(\Sigma)$ is freely generated by all $\alpha_{i}$ and $\beta_{i}$. Here $\Sigma$ is the bottom surface stage of the grope $(G, \gamma)$ with a standard symplectic basis of circles $\alpha_{i}, \beta_{i}$. The Magnus expansion for the free Milnor group (see [10], [5] or the proof of Theorem 3) shows that $\gamma=\prod\left[\alpha_{i}, \beta_{i}\right]$ does not lie in $M \pi_{1}(\Sigma)^{3}$. Similarly, for $k>2, \pi_{1}(G)$ is freely generated by those circles in a standard symplectic basis of a surface stage in $G$ to which nothing else is attached. Now assume that the $k$-grope $(G, \gamma)$ is obtained by attaching $p_{i}$-gropes $G_{\alpha_{i}}$ to $\alpha_{i}$ and $q_{i}$-gropes $G_{\beta_{i}}$ to $\beta_{i}, p_{i}+q_{i} \geq k$. By induction, $\alpha_{i} \notin M \pi_{1}\left(G_{\alpha_{i}}\right)^{p_{i}+1}$ and $\beta_{i} \notin M \pi_{1}\left(G_{\beta_{i}}\right)^{q_{i}+1}$ since $p_{i}, q_{i} \geq 1$. But the free generators of $\pi_{1}\left(G_{\alpha_{i}}\right)$ and $\pi_{1}\left(G_{\beta_{i}}\right)$ are contained in the set of free generators of $\pi_{1}(G)$ and therefore $\gamma=\prod\left[\alpha_{i}, \beta_{i}\right] \notin M \pi_{1}(G)^{k+1}$. Again, this may be seen by applying the Magnus expansion to $M \pi_{1}(G)$.

Remark In the case when all stages of a Grope $X$ are tori, the correspondence between its tree $T_{X}$ and the trees of the dual Gropes, given in the proof of theorem 1, is particularly appealing and easy to describe. Let $\gamma$ be a tip of $T_{X}$. The tree for the Grope, Alexander-dual to $\gamma$, is obtained by redrawing $T_{X}$, only with $\gamma$ drawn as the root, see Figure 6 .
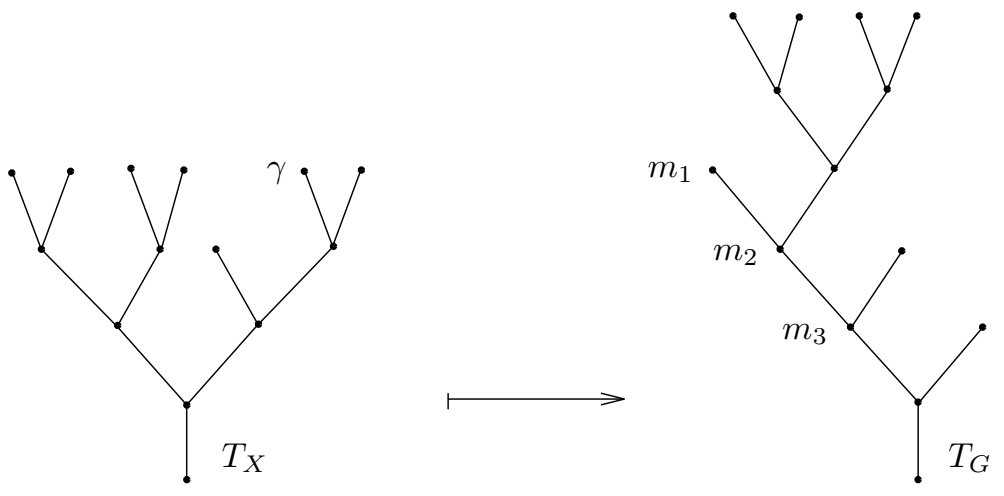

Figure 6: Tree duality in the genus 1 case

As a corollary of Theorem 1 we get the following result.

Corollary 4.2 Let $L=\left(l_{1}, \ldots, l_{n}\right)$ and $L^{\prime}=\left(l_{1}^{\prime}, \ldots, l_{n}^{\prime}\right)$ be two links in $S^{3} \times$ $\{0\}$ and $S^{3} \times\{1\}$ respectively. Suppose there are disjointly embedded annuluslike Gropes $A_{1}, \ldots, A_{n}$ of class $k$ in $S^{3} \times[0,1]$ with $\partial A_{i}=l_{i} \cup l_{i}^{\prime}, i=1, \ldots, n$. Then there is an isomorphism of nilpotent quotients

$$
\pi_{1}\left(S^{3} \backslash L\right) / \pi_{1}\left(S^{3} \backslash L\right)^{k} \cong \pi_{1}\left(S^{3} \backslash L^{\prime}\right) / \pi_{1}\left(S^{3} \backslash L^{\prime}\right)^{k}
$$


Remark For those readers who are familiar with Milnor's $\bar{\mu}$-invariants we should mention that the above statement directly implies that for any multiindex $I$ of length $|I| \leq k$ one gets $\bar{\mu}_{L}(I)=\bar{\mu}_{L^{\prime}}(I)$. For a different proof of this consequence see [8].

Proof of Corollary 4.2 The proof is a $\phi_{k}$-version of Stallings' proof of the concordance invariance of all nilpotent quotients of $\pi_{1}\left(S^{3} \backslash L\right)$, see [12]. Namely, Alexander duality and Theorem 1 imply that the inclusion maps

$$
\left(S^{3} \times\{0\} \backslash L\right) \hookrightarrow\left(S^{3} \times[0,1] \backslash\left(A_{1} \cup \ldots \cup A_{n}\right)\right) \hookleftarrow\left(S^{3} \times\{1\} \backslash L^{\prime}\right)
$$

induce isomorphisms on $H_{1}($.$) and on H_{2}(.) / \phi_{k}$. So by Dwyer's Theorem they induce isomorphisms on $\pi_{1} /\left(\pi_{1}\right)^{k}$.

\section{The Link Composition Lemma}

The Link Composition Lemma was originally formulated in [2]. The reader should be cautious about its proof given there; it can be made precise using Milnor's $\bar{\mu}$-invariants with repeating coefficients, while this section presents an alternative proof.

Given a link $\widehat{L}=\left(l_{1}, \ldots, l_{k+1}\right)$ in $S^{3}$ and a link $Q=\left(q_{1}, \ldots, q_{m}\right)$ in the solid torus $S^{1} \times D^{2}$, their "composition" is obtained by replacing the last component of $\widehat{L}$ with $Q$. More precisely, it is defined as $L \cup \phi(Q)$ where $L=\left(l_{1}, \ldots, l_{k}\right)$ and $\phi: S^{1} \times D^{2} \hookrightarrow S^{3}$ is a 0 -framed embedding whose image is a tubular neighborhood of $l_{k+1}$. The meridian $\{1\} \times \partial D^{2}$ of the solid torus will be denoted by $\wedge$ and we put $\widehat{Q}:=Q \cup \wedge$. We sometimes think of $Q$ or $\widehat{Q}$ as links in $S^{3}$ via the standard embedding $S^{1} \times D^{2} \hookrightarrow S^{3}$.

Theorem 3 (Link Composition Lemma)

(i) If $\widehat{L}$ and $\widehat{Q}$ are both homotopically essential in $S^{3}$ then $L \cup \phi(Q)$ is also homotopically essential.

(ii) Conversely, if $L \cup \phi(Q)$ is homotopically essential and if both $\widehat{L}$ and $\widehat{Q}$ are almost homotopically trivial, then both $\widehat{L}$ and $\widehat{Q}$ are homotopically essential in $S^{3}$.

Remark Part (ii) does not hold without the almost triviality assumption on $\widehat{L}$ and $\widehat{Q}$. For example, let $\widehat{L}$ consist of just one component $l_{1}$, and let $Q$ be a Hopf link contained in a small ball in $S^{1} \times D^{2}$. Then $L \cup \phi(Q)=\phi(Q)$ is homotopically essential, yet $\widehat{L}$ is trivial. 


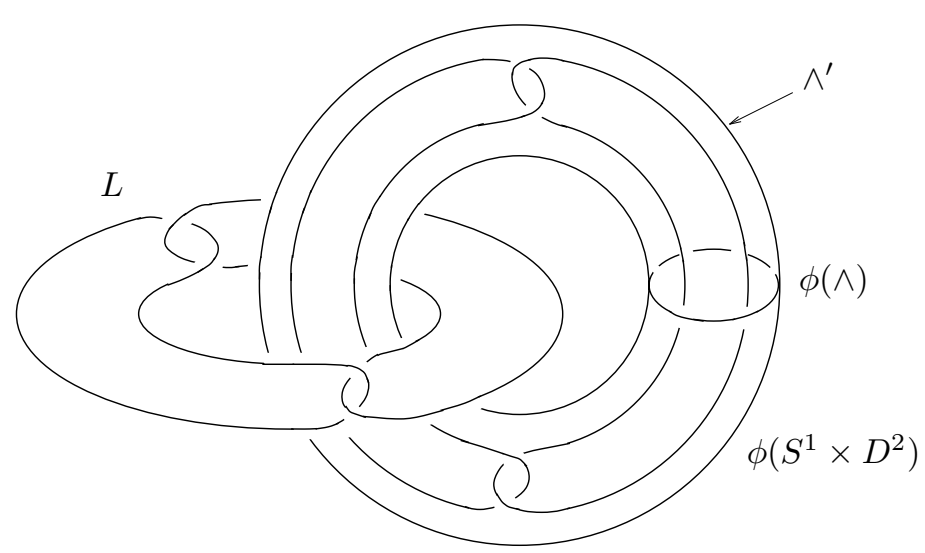

Figure 7: In this example $\widehat{L}$ is the Borromean rings, and $Q$ is the Bing double of the core circle of $S^{1} \times D^{2}$.

In part (i), if either $L$ or $Q$ is homotopically essential, then their composition $L \cup \phi(Q)$ is also essential. (Note that $\widehat{Q}$ and $\phi(\widehat{Q})$ are homotopically equivalent, see Lemma 3.2 in [2].) If neither $L$ nor $Q$ is homotopically essential, then by deleting some components of $L$ and $Q$ if necessary, one may assume that $\widehat{L}$ and $\widehat{Q}$ are almost homotopically trivial (and still homotopically essential). In the case when $L \cup \phi(Q)$ is not almost homotopically trivial part (i) follows immediately. Similarly, part (ii) can be proved in this case easily by induction on the number of components of $L$ and $Q$.

Therefore, we will assume from now on that $\widehat{L}, \widehat{Q}$ and $L \cup \phi(Q)$ are almost homotopically trivial links in $S^{3}$.

Lemma 5.1 If $\widehat{L}$ and $\widehat{Q}$ are both homotopically trivial in $S^{3}$ then $\phi(\wedge)$ represents the trivial element in the Milnor group $M(L \cup \phi(Q))$.

Proof Let $\wedge^{\prime}$ denote $\phi\left(S^{1} \times\{1\}\right)$. The Milnor group $M(L \cup \phi(Q))$ is nilpotent of class $k+m+1$, so it suffices to show that $\phi(\wedge)$ represents an element in $\pi_{1}\left(S^{3} \backslash(L \cup \phi(Q))\right)^{k+m+1}$. This will be achieved by constructing an $\infty$-grope $G$ bounded by $\phi(\wedge)$ in the complement of $L \cup \phi(Q)$. In fact, the construction also gives an $\infty$-grope $G^{\prime} \subset S^{3} \backslash(L \cup \phi(Q))$ bounded by $\wedge^{\prime}$.

Consider $S^{1} \times D^{2}$ as a standard unknotted solid torus in $S^{3}$, and let $c$ denote the core of the complementary solid torus $D^{2} \times S^{1}$. Since $\widehat{Q}$ is homotopically trivial, after changing $Q$ by an appropriate link homotopy in $S^{1} \times D^{2}, \wedge$ bounds 
an immersed disk $\Delta \subset S^{3}$ in the complement of the new link. Denote the new link by $Q$ again. Similarly $L$ can be changed so that the untwisted parallel copy $\wedge^{\prime}$ of $l_{k+1}$ bounds a disk $\Delta^{\prime} \subset S^{3} \backslash L$. Recall that $M(L \cup \phi(Q))$ does not change if $L \cup \phi(Q)$ is modified by a link homotopy.

The intersection number of $\Delta$ with $c$ is trivial, since $\wedge$ and $c$ do not link. Replace the union of disks $\Delta \cap\left(D^{2} \times S^{1}\right)$ by annuli lying in $\partial\left(D^{2} \times S^{1}\right)$ to get $\Sigma \subset S^{1} \times D^{2} \backslash Q$, an immersed surface bounded by $\wedge$. Similarly the intersection number of $\Delta^{\prime}$ with the core circle of $\phi\left(S^{1} \times D^{2}\right)$ is trivial, and $\wedge^{\prime}$ bounds $\Sigma^{\prime} \subset S^{3} \backslash(L \cup \phi(Q))$. The surfaces $\phi(\Sigma)$ and $\Sigma^{\prime}$ are the first stages of the gropes $G$ and $G^{\prime}$ respectively.

Notice that half of the basis for $H_{1}(\phi(\Sigma))$ is represented by parallel copies of $\wedge^{\prime}$. They bound the obvious surfaces: annuli connecting them with $\wedge^{\prime}$ union with $\Sigma^{\prime}$, which provide the second stage for $G$. Since this construction is symmetric, it provides all higher stages for both $G$ and $G^{\prime}$.

Lemma 5.2 Let $i$ : $S^{3} \backslash$ neighborhood $\left(\widehat{L} \backslash l_{1}\right) \longrightarrow S^{3} \backslash\left(L \cup \phi(Q) \backslash l_{1}\right)$ denote the inclusion map, and let $i_{\#}$ be the induced map on $\pi_{1}$. Then $i_{\#}$ induces a well defined map $i_{*}$ of Milnor groups.

Remark Given two groups $G$ and $H$ normally generated by $g_{i}$ respectively $h_{j}$, let $M G$ and $M H$ be their Milnor groups defined with respect to the given sets of normal generators. If a homomorphism $\phi: G \longrightarrow H$ maps each $g_{i}$ to one of the $h_{j}$ then it induces a homomorphism $M \phi: M G \longrightarrow M H$. In general, $\phi: G \longrightarrow H$ induces a homomorphism of the Milnor groups if and only if $\phi\left(g_{i}\right)$ commutes with $\phi\left(g_{i}\right)^{\phi(g)}$ in $M H$ for all $i$ and all $g \in G$.

Proof of Lemma 5.2 The Milnor groups $M\left(\widehat{L} \backslash l_{1}\right)$ and $M\left(L \cup \phi(Q) \backslash l_{1}\right)$ are generated by meridians. Moreover, $i_{\#}\left(m_{i}\right)=m_{i}$ for $i=2, \ldots, k$ and $i_{\#}\left(m_{k+1}\right)=\phi(\wedge)$ where $m_{1}, \ldots, m_{k+1}$ are meridians to the components of $\widehat{L}$. Hence to show that $i_{*}$ is well-defined it suffices to prove that all the commutators

$$
\left[\phi(\wedge),(\phi \wedge)^{i_{\#}(g)}\right], \quad g \in \pi_{1}\left(S^{3} \backslash\left(\widehat{L} \backslash l_{1}\right)\right),
$$

are trivial in $\left.M\left(L \cup \phi(Q) \backslash l_{1}\right)\right)$. Consider the following exact sequence, obtained by deleting the component $q_{1}$ of $Q$.

$$
\operatorname{ker}(\psi) \longrightarrow M\left(L \cup \phi(Q) \backslash l_{1}\right) \stackrel{\psi}{\longrightarrow} M\left(L \cup \phi(Q) \backslash\left(l_{1} \cup \phi\left(q_{1}\right)\right)\right) \longrightarrow 0
$$


An application of Lemma 5.1 to $\left(\widehat{L} \backslash l_{1}\right)$ and to $\left(\widehat{Q} \backslash q_{1}\right)$ shows that $\psi(\phi(\wedge))=1$ and hence $\phi(\wedge), \phi(\wedge)^{g} \in \operatorname{ker}(\psi)$. The observation that $\operatorname{ker}(\psi)$ is generated by the meridians to $\phi\left(q_{1}\right)$ and hence is commutative finishes the proof of Lemma 5.2.

Proof of Theorem 3 Let $M\left(F_{m_{1}, \ldots, m_{s+1}}\right)$ be the Milnor group of a free group, ie the Milnor group of the trivial link on $s+1$ components with meridians $m_{i}$. Let $R\left(y_{1}, \ldots, y_{s}\right)$ be the quotient of the free associative ring on generators $y_{1}, \ldots, y_{s}$ by the ideal generated by the monomials $y_{i_{1}} \cdots y_{i_{r}}$ with one index occurring at least twice. The additive group $\left(R\left(y_{1}, \ldots, y_{s}\right),+\right)$ of this ring is free abelian on generators $y_{i_{1}} \cdots y_{i_{r}}$ where all indices are distinct. Milnor [10] showed that setting $m_{s+1}=1$ induces a short exact sequence of groups

$$
1 \longrightarrow\left(R\left(y_{1}, \ldots, y_{s}\right),+\right) \stackrel{r}{\longrightarrow} M\left(F_{m_{1}, \ldots, m_{s+1}}\right) \stackrel{i}{\longrightarrow} M\left(F_{m_{1}, \ldots, m_{s}}\right) \longrightarrow 1
$$

where $r$ is defined on the above free generators by left-iterated commutators with $m_{s+1}$ :

$$
r\left(y_{j_{1}} \cdots y_{j_{k}}\right):=\left[m_{j_{1}},\left[m_{j_{2}}, \ldots,\left[m_{j_{k}}, m_{s+1}\right] \ldots\right]\right]
$$

In particular, $r(0)=1$ and $r(1)=m_{s+1}$. Obviously, the above extension of groups splits by sending $m_{i}$ to $m_{i}$. This splitting induces the following conjugation action of $M\left(F_{m_{1}, \ldots, m_{s}}\right)$ on $R\left(y_{1}, \ldots, y_{s}\right)$. Let $Y:=y_{j_{1}} \cdots y_{j_{k}}$, then

$$
\begin{gathered}
m_{i} \cdot r(Y) \cdot m_{i}^{-1}=\left[m_{i}, r(Y)\right] \cdot r(Y)= \\
{\left[m_{i},\left[m_{j_{1}},\left[m_{j_{2}}, \ldots,\left[m_{j_{k}}, m_{s+1}\right] \ldots\right]\right] \cdot r(Y)=r\left(\left(y_{i}+1\right) \cdot Y\right)\right.}
\end{gathered}
$$

which implies that $m_{i}$ acts on $R\left(y_{1}, \ldots, y_{s}\right)$ by ring multiplication with $y_{i}+1$ on the left. Since $m_{i}$ generate the group $M\left(F_{m_{1}, \ldots, m_{s}}\right)$ this defines a well defined homomorphism of $M\left(F_{m_{1}, \ldots, m_{s}}\right)$ into the units of the ring $R\left(y_{1}, \ldots\right.$, $\left.y_{s}\right)$. In fact, this is the Magnus expansion, well known in the context of free groups (rather than free Milnor groups). We conclude in particular, that the abelian group $\left(R\left(y_{1}, \ldots, y_{s}\right),+\right)$ is generated by $y_{i}$ as a module over the group $M\left(F_{m_{1}, \ldots, m_{s}}\right)$.

Returning to the notation of Theorem 3, we have the following commutative diagram of group extensions. We use the fact that the links $L \cup \phi(Q) \backslash l_{1}$ and $\widehat{L} \backslash l_{1}$ are homotopically trivial. Here $y_{i}$ are the variables corresponding to the link $L$ and $z_{j}$ are the variables corresponding to $\phi(Q)$. We introduce short notations $R(\mathcal{Y}):=R\left(y_{1}, \ldots, y_{k}\right)$ and $R(\mathcal{Y}, \mathcal{Z}):=R\left(y_{1}, \ldots, y_{k}, z_{2}, \ldots, z_{m}\right)$. 


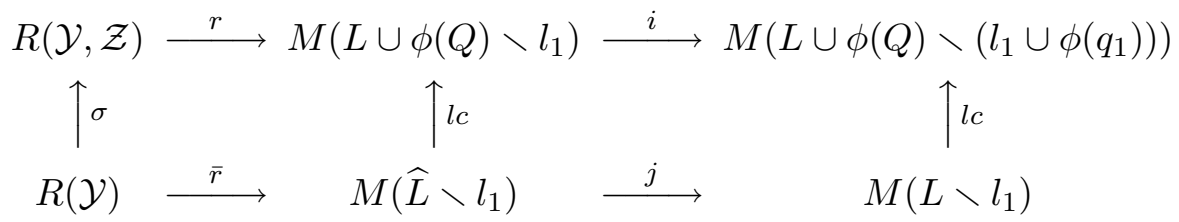

Recall that by definition $l c\left(m_{i}\right)=m_{i}$ for all meridians $m_{2}, \ldots, m_{k}$ of $L \backslash l_{1}$. Moreover, the link composition map $l c$ sends the meridian $m_{k+1}$ to the $\wedge$-curve of $\phi(Q)$.

The existence of the homomorphism $l c$ on the Milnor group level already implies our claim (ii) in Theorem 3: By assumption, $l_{1}$ represents the trivial element in $M\left(\widehat{L} \backslash l_{1}\right)$ since $\widehat{L}$ is homotopically trivial. Consequently, $l c\left(l_{1}\right)=l_{1}$ is also trivial in $M\left(L \cup \phi(Q) \backslash l_{1}\right)$ and hence by [10] the link $L \cup \phi(Q)$ is homotopically trivial.

The key fact in our approach to part (i) of Theorem 3 is the following result which says that link composition corresponds to ring multiplication.

Lemma 5.3 The homomorphism $\sigma: R\left(y_{2}, \ldots, y_{k}\right) \longrightarrow R\left(y_{2}, \ldots, y_{k}, z_{2}, \ldots, z_{m}\right)$ is given by ring multiplication with $r^{-1}(\wedge)$ on the right.

Note that by Lemma $5.1 \wedge$ is trivial in $M\left(L \cup \phi(Q) \backslash\left(l_{1} \cup \phi\left(q_{1}\right)\right)\right)$, so that it makes sense to consider $r^{-1}(\wedge)$. We will abbreviate this important element by $\wedge_{R}$.

Proof of Lemma 5.3 Since the above diagram commutes and $\left(R\left(y_{2}, \ldots, y_{k}\right)\right.$, $+)$ is generated by $y_{i}$ as a module over the group $M\left(F_{m_{2}, \ldots, m_{k}}\right)$ it suffices to check our claim for these generators $y_{i}$. We get by definition

$$
\begin{aligned}
l c\left(\bar{r}\left(y_{i}\right)\right)= & l c\left(\left[m_{i}, m_{k+1}\right]\right)=\left[m_{i}, \wedge\right]=\left(m_{i} \cdot \wedge \cdot m_{i}^{-1}\right) \cdot \wedge^{-1} \\
& =r\left(\left(y_{i}+1\right) \cdot \wedge_{R}\right) \cdot \wedge^{-1}=r\left(y_{i} \cdot \wedge_{R}\right) .
\end{aligned}
$$

We are using the fact that conjugation by $m_{i}$ corresponds to left multiplication by $\left(y_{i}+1\right)$.

Since $L$ is homotopically trivial and $\widehat{L}$ is homotopically essential, it follows that $0 \neq l_{1} \in \operatorname{ker}(j)$. After possibly reordering the $y_{i}$ this implies in addition that for some integer $a \neq 0$ we have

$$
\bar{r}^{-1}\left(l_{1}\right)=a \cdot\left(y_{2} \cdots y_{k}\right)+\text { terms obtained by permutations from } y_{2} \cdots y_{k} \text {. }
$$


Setting all the meridians $m_{i}$ of $L$ to 1 (which implies setting the variables $y_{i}$ to 0 ), we get a commutative diagram of group extensions

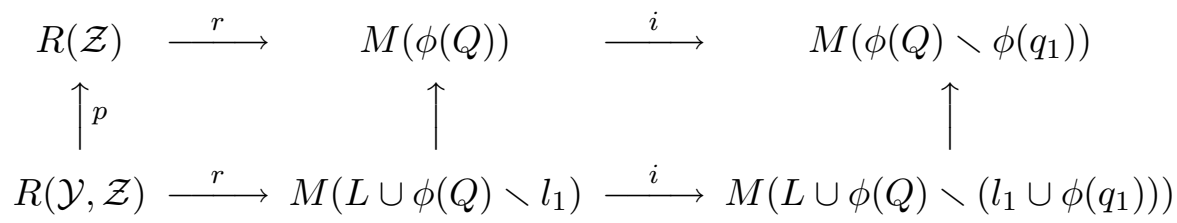

As before, $R(\mathcal{Z})$ and $R(\mathcal{Y}, \mathcal{Z})$ are short notations for $R\left(z_{2}, \ldots, z_{m}\right)$ and $R\left(y_{1}\right.$, $\left.\ldots, y_{k}, z_{2}, \ldots, z_{m}\right)$ respectively. Since $\widehat{Q}$ (and equivalently $\left.\phi(\widehat{Q})\right)$ is homotopically essential we have $0 \neq \wedge \in \operatorname{ker}(i)$. This shows that $p\left(\wedge_{R}\right) \neq 0$. The almost triviality of $\widehat{Q}$ implies in addition that after possibly reordering the $z_{j}$ we have for some integer $b \neq 0$

$$
p\left(\wedge_{R}\right)=b \cdot\left(z_{2} \cdots z_{m}\right)+\text { terms obtained by permutations from } z_{2} \cdots z_{m} .
$$

It follows from Lemma 5.3 that $r^{-1}\left(l_{1}\right)=\bar{r}^{-1}\left(l_{1}\right) \cdot \wedge_{R}$. This product contains the term

$$
a b \cdot\left(y_{2} \cdots y_{k} \cdot z_{2} \cdots z_{m}\right)
$$

the coefficient $a b$ of which is non-zero. This completes the proof of Theorem 3 .

Remark Those readers who are familiar with Milnor's $\bar{\mu}$-invariants will have recognized that the above proof in fact shows that the first non-vanishing $\bar{\mu}-$ invariants are multiplicative under link composition. 


\section{References}

[1] W Dwyer, Homology, Massey products and maps between groups, J. Pure Appl. Algebra 6 (1975) 177-190

[2] MH Freedman, X S Lin, On the $(A, B)$-slice problem, Topology 28 (1989) 91-110

[3] MH Freedman, F Quinn, The topology of 4-manifolds, Princeton Math. Series 39, Princeton, NJ (1990)

[4] M H Freedman, P Teichner, 4-manifold topology I: subexponential groups, Invent. Math. 122 (1995) 509-529

[5] M H Freedman, P Teichner, 4-manifold topology II: Dwyer's filtration and surgery kernels, Invent. Math. 122 (1995) 531-557

[6] C Giffen, Link concordance implies link homotopy, Math. Scand. 45 (1979) 243-254

[7] D Goldsmith, Concordance implies homotopy for classical links in $S^{3}$, Comment. Math. Helvitici 54 (1979) 347-355

[8] V S Krushkal, Additivity properties of Milnor's $\bar{\mu}$-invariants, (to appear)

[9] X S Lin, On equivalence relations of links in 3-manifolds, preprint (1985)

[10] J Milnor, Link Groups, Annals of Math. 59 (1954) 177-195

[11] J Milnor, Isotopy of links, Algebraic geometry and topology, Princeton Univ. Press (1957) 280-306

[12] J Stallings, Homology and central series of groups, J. Algebra 2 (1965) 19701981

[13] P Teichner, Stratified Morse Theory and Link Homotopy, Habilitationsschrift (in preparation) 